\title{
A simple algorithm for the Kohn-Sham inversion problem applicable to general target densities
}

\author{
Kati Finzel · Paul W. Ayers · Patrick Bultinck
}

Received: 0 September 2009 / Accepted: 0 September 2009

\begin{abstract}
A simple algorithm for the Kohn-Sham inversion problem is presented. The method is found to converge towards a nearby $v$-representable Kohn-Sham density irrespective of the fact whether the initial target density has been $v$ representable or not. For the proposed procedure the target density can be of general nature. The algorithm can handle Hartree-Fock and post Hartree-Fock, spin-unpolarized and polarized states equally well. Additionally, experimental densities and even general gedanken densities can be treated. The algorithm is easy to implement and does not require an additional procedure to adjust eigenvalues.
\end{abstract}

Keywords Kohn-Sham potential · Kohn-Sham inversion procedure $\cdot$ target density $\cdot$ gedanken densities

\section{INTRODUCTION}

The most prominent method for practical density functional theory (DFT) [1, 2] calculations is the Kohn-Sham (KS) method, in which the electron density is determined by solving a set of single-particle equations with an effective one-body potential. The KS method requires an external input, namely the exchange-correlation potential, as part of this effective one-body potential. The exchange-correlation potential is defined as the functional derivative of the exchange-correlation energy, which accounts for all non-classical many-body effects of the electronic interaction. Since the Hohenberg-Kohn

\section{Kati Finzel}

Department of Inorganic and Physical Chemistry, Ghent University, Krijgslaan 281 (S3), 9000 Ghent, Belgium E-mail: Kati.Finzel@UGent.be

Paul W. Ayers

Department of Chemistry and Chemical Biology, McMaster University, Hamilton, ON L8S 4M1, Canada

Patrick Bultinck

Department of Inorganic and Physical Chemistry, Ghent University, Krijgslaan 281 (S3), 9000 Ghent, Belgium theorems [3] only prove the existence of such an effective one-body potential, but gives no clues on how to effectively construct it, there is large interest in effective methods how to obtain the KS potential from wavefunction based calculations in order to improve functional design in a systematic manner.

For this so-called inversion problem several algorithms have been suggested and applied in the recursive construction of exchange-correlation functionals [4-24]. However, some methods are restricted to rather small systems or specific wavefunction types like Hartree-Fock (HF) theory, some of them are numerically unstable or difficult to implement or they require additional empirical parameters. Recently, an algorithm solving problems concerning basis set instabilities was presented by Staroverov et al. [24]. Here, a new, simple and elusive algorithm for the KS inversion problem is presented. The method is found to converge towards $v$-representable electron density that is close to the chosen target density. The target density is general. The method can equally well be applied to uncorrelated and correlated wavefunction based densities as well as experimental densities and even gedanken densities[25]. Additionally, spinpolarization is supported. The proposed method is free from empirical parameters and does not require an additional procedure for adjusting the eigenvalues during the iterative process.

\section{THEORY}

The KS inversion procedure deals with the problem how to obtain that effective local potential $v_{\mathrm{eff}}([\rho] ; \mathbf{r})[26]$ :

$$
-\frac{1}{2} \nabla^{2} \Phi_{i}(\mathbf{r})+v_{\mathrm{eff}}([\rho] ; \mathbf{r}) \Phi_{i}(\mathbf{r})=\varepsilon_{i} \Phi_{i}(\mathbf{r})
$$

such that the resulting squared occupied eigenfunctions $\Phi_{i}(\mathbf{r})$ with $\varepsilon_{i}$ eigenvalues sum up to the desired electron density 
$\rho(\mathbf{r}):$

$\rho(\mathbf{r})=\sum_{i}^{\mathrm{occ}}\left|\Phi_{i}(\mathbf{r})\right|^{2}$.

For the proposed method the target density $\rho^{(\operatorname{tar})}(\mathbf{r})$ is of general nature, as only numerical data stored, for example, on a grid is needed. Thus, the algorithm handles equally well spin-unpolarized as well as spin-polarized HF and post-HF electron densities, but also experimental densities and even gedanken densities can be handled by the following procedure determining the effective local potential for a given target density. Usually, the effective local potential is split into the nuclear potential $v_{Z}([\rho] ; \mathbf{r})$, the Hartree potential $v_{\mathrm{H}}([\rho] ; \mathbf{r})$, and the exchange-correlation potential $v_{X C}([\rho] ; \mathbf{r})$;

$v_{\mathrm{eff}}([\rho] ; \mathbf{r})=v_{Z}([\rho] ; \mathbf{r})+v_{\mathrm{H}}([\rho] ; \mathbf{r})+v_{X C}([\rho] ; \mathbf{r})$.

Hereby, $v_{Z}([\rho] ; \mathbf{r})$ and $v_{\mathrm{H}}([\rho] ; \mathbf{r})$ are known as explicit density functionals:

$v_{\mathrm{H}}([\rho] ; \mathbf{r})=\int \frac{\rho\left(\mathbf{r}^{\prime}\right)}{\left|\mathbf{r}^{\prime}-\mathbf{r}\right|} \mathrm{d} \mathbf{r}^{\prime}$

and:

$v_{Z}([\rho] ; \mathbf{r})=-\sum_{A}^{M} \frac{Z_{A}}{\left|\mathbf{R}_{A}-\mathbf{r}\right|}$

for a system of $M$ nuclei with charge $Z_{A}$ located at $\mathbf{R}_{A}$. (Strictly speaking, Eq. 5 is only true if the density is obtained by an exact (i.e., full-CI) calculation in the basis set limit. Eq. 5 should be an accurate approximation for accurate calculations, however.) Thus, the exchange-correlation potential $v_{X C}([\rho] ; \mathbf{r})$ remains the only unknown term which needs to be determined. At the solution point, the Euler equation is fulfilled:[3]

$$
\begin{array}{r}
0=v_{\mathrm{W}}([\rho] ; \mathbf{r})+v_{\mathrm{P}}([\rho] ; \mathbf{r})+v_{Z}([\rho] ; \mathbf{r}) \\
+v_{\mathrm{H}}([\rho] ; \mathbf{r})+v_{X C}([\rho] ; \mathbf{r})-\mu
\end{array}
$$

where $\mu$ is the chemical potential [2] introduced as Lagrange multiplier for an electron density normalized to $N$ electrons $\left(0=\mu\left[\int \rho(\mathbf{r}) \mathrm{d} \mathbf{r}-N\right]\right)$. The von Weizsäcker potential[27] $v_{\mathrm{W}}([\rho] ; \mathbf{r})$ and the Pauli potential $[28] v_{\mathrm{P}}([\rho] ; \mathbf{r})$ originate from the kinetic energy. While the von Weizsäcker potential is known as an explicit density functional:

$\nu_{\mathrm{W}}([\rho] ; \mathbf{r})=\frac{1}{8} \frac{(\nabla \rho(\mathbf{r}))^{2}}{\rho^{2}(\mathbf{r})}-\frac{1}{4} \frac{\nabla^{2} \rho(\mathbf{r})}{\rho(\mathbf{r})}$,

the Pauli potential is not known as an explicit functional of the electron density. For the KS system the Pauli potential can formally be expressed with the help of the eigenfunctions $\Phi_{i}(\mathbf{r})$ and eigenvalues $\varepsilon_{i}$ [29]:

$\nu_{\mathrm{P}}([\rho] ; \mathbf{r})=\frac{\tau(\mathbf{r})-t_{\mathrm{W}}(\mathbf{r})}{\rho(\mathbf{r})}+\sum_{i}^{\mathrm{occ}}\left(\mu-\varepsilon_{i}\right) \frac{\left|\Phi_{i}(\mathbf{r})\right|^{2}}{\rho(\mathbf{r})}$ where for an effective potential asymptotically decaying to zero $\mu$ equals the highest occupied eigenvalue $\varepsilon_{M}, \tau(\mathbf{r})=$ $1 / 2 \sum_{i}\left|\nabla \Phi_{i}(\mathbf{r})\right|^{2}$ is the positive kinetic energy density and $t_{\mathrm{W}}(\mathbf{r})=1 / 8|\nabla \rho(\mathbf{r})|^{2} / \rho(\mathbf{r})$ is the Weizsäcker kinetic energy density.

The following iterative procedure is proposed: All explicit density functionals are evaluated from the target density $\rho^{(\text {tar) }}$. A convenient starting point for $\nu_{X C}([\rho] ; \mathbf{r})$ is the Fermi-Amaldi model [30] first suggested in this context by Parr and coworkers $[10,31]$. Then, the Pauli potential $\nu_{\mathrm{P}}^{(n)}\left(\left[\rho^{(n)}\right] ; \mathbf{r}\right)$ is evaluated from the actual eigenfunctions and eigenvalues of the $n$-th step, cf. Eq. 8, yielding the new exchangecorrelation potential $v_{X C}^{(n+1)}\left(\left[\rho^{(n)}\right] ; \mathbf{r}\right)$ :

$$
\begin{array}{r}
v_{X C}^{(n+1)}\left(\left[\rho^{(n)}\right] ; \mathbf{r}\right)=\mu^{n}-v_{\mathrm{W}}\left(\left[\rho^{(\operatorname{tar})}\right] ; \mathbf{r}\right)-v_{\mathrm{P}}^{(n)}\left(\left[\rho^{(n)}\right] ; \mathbf{r}\right) \\
-v_{Z}\left(\left[\rho^{(\operatorname{tar})}\right] ; \mathbf{r}\right)-v_{\mathrm{H}}\left(\left[\rho^{(\operatorname{tar})}\right] ; \mathbf{r}\right) .
\end{array}
$$

The process is repeated until convergence. A convenient convergence criterion is , for example, the distance $d_{p}\left(\rho^{(n+1)}, \rho^{(n)}\right)$ [32] between two consecutive steps:

$d_{p}\left(\rho^{(n+1)}, \rho^{(n)}\right)=\sqrt[p]{\int\left|\rho^{(n+1)}(\mathbf{r})-\rho^{(n)}(\mathbf{r})\right|^{p} \mathrm{~d} \mathbf{r}}$

or alternatively, the distance from the target density $d_{p}\left(\rho^{(n)}, \rho^{(\operatorname{tar})}\right)$.

The proposed algorithm can be reformulated in the spirit of a density-based update on a given trial potential, similar to the methods proposed in references [12, 20]. To see that, replace $v_{\mathrm{P}}^{(n)}(\{\Phi(\mathbf{r})\} ; \mathbf{r})$ in Eq. 9 by Eq. 8 , make use of the fact that the actual eigenfunctions obey Eq. 1 obtained from the previous exchange-correlation potential $v_{X C}^{(n)}\left(\left[\rho^{(n-1)}\right] ; \mathbf{r}\right)$, recall that all density-based potentials are evaluated from the target density $\rho(\mathbf{r})$ and rearrange to get:

$$
\begin{array}{r}
v_{X C}^{(n+1)}\left(\left[\rho^{(n)}\right] ; \mathbf{r}\right)=v_{X C}^{(n)}\left(\left[\rho^{n-1}\right] ; \mathbf{r}\right)-v_{\mathrm{W}}\left(\left[\rho^{(\operatorname{tar})}\right] ; \mathbf{r}\right) \\
+v_{\mathrm{W}}\left(\left[\rho^{(n)}\right] ; \mathbf{r}\right) \\
=v_{X C}^{(n)}\left(\left[\rho^{(n-1)}\right] ; \mathbf{r}\right)- \\
-\frac{1}{8} \frac{\left(\nabla \rho^{(\operatorname{tar})}(\mathbf{r})\right)^{2}}{\left(\rho^{(\operatorname{tar})}(\mathbf{r})\right)^{2}}+\frac{1}{4} \frac{\nabla^{2} \rho^{(\operatorname{tar})}(\mathbf{r})}{\rho^{(\operatorname{tar})}(\mathbf{r})} \\
+\frac{1}{8} \frac{\left(\nabla \rho^{(n)}(\mathbf{r})\right)^{2}}{\left(\rho^{(n)}(\mathbf{r})\right)^{2}}-\frac{1}{4} \frac{\nabla^{2} \rho^{(n)}(\mathbf{r})}{\rho^{(n)}(\mathbf{r})} .
\end{array}
$$

In contrast to the previously mentioned methods[12, 20] using an approximate update based on the density difference or the density ratio, the update in the above Eq. 11 results from the sum of the Euler equations for the actual trial density and the target electron density. To see that, recall that the Euler equation for the target density is:

$$
\begin{aligned}
0=v_{\mathrm{W}} & \left(\left[\rho^{(\mathrm{tar})}\right] ; \mathbf{r}\right)+v_{\mathrm{P}}\left(\left[\rho^{(\mathrm{tar})}\right] ; \mathbf{r}\right)+v_{Z}\left(\left[\rho^{(\mathrm{tar})}\right] ; \mathbf{r}\right) \\
& +v_{\mathrm{H}}\left(\left[\rho^{(\mathrm{tar})}\right] ; \mathbf{r}\right)+v_{X C}\left(\left[\rho^{(\mathrm{tar})}\right] ; \mathbf{r}\right)-\mu^{(\mathrm{tar})} .
\end{aligned}
$$

The corresponding Euler equation for the actual density $\rho^{(n)}$ fulfilled in the $n$-the step is given by:

$$
\begin{array}{r}
0=v_{\mathrm{W}}\left(\left[\rho^{(n)}\right] ; \mathbf{r}\right)+v_{\mathrm{P}}\left(\left[\rho^{(n)}\right] ; \mathbf{r}\right)+v_{Z}\left(\left[\rho^{(n)}\right] ; \mathbf{r}\right) \\
+v_{\mathrm{H}}\left(\left[\rho^{(n)}\right] ; \mathbf{r}\right)+v_{X C}\left(\left[\rho^{(n)}\right] ; \mathbf{r}\right)-\mu^{(n)} .
\end{array}
$$


Sum Eqs. 12 and 13 and rearrange to see that the requirement that the Euler equation shall be fulfilled for the actual Pauli potential in combination with the remaining density based potentials evaluated from the target density:

$$
\begin{array}{r}
0=v_{\mathrm{W}}\left(\left[\boldsymbol{\rho}^{(\mathrm{tar})}\right] ; \mathbf{r}\right)+v_{\mathrm{P}}\left(\left[\rho^{(n)}\right] ; \mathbf{r}\right)+v_{Z}\left(\left[\rho^{(\operatorname{tar})}\right] ; \mathbf{r}\right) \\
+v_{\mathrm{H}}\left(\left[\rho^{(\operatorname{tar})}\right] ; \mathbf{r}\right)+v_{X C}^{(n+1)}\left(\left[\rho^{(n)}\right] ; \mathbf{r}\right)-\mu^{(n)}
\end{array}
$$

yields the new exchange-correlation potential:

$$
\begin{gathered}
v_{X C}^{(n+1)}\left(\left[\rho^{(n)}\right] ; \mathbf{r}\right)=v_{\mathrm{W}}\left(\left[\rho^{(n)}\right] ; \mathbf{r}\right)+v_{\mathrm{P}}\left(\left[\rho^{(\operatorname{tar})}\right] ; \mathbf{r}\right)+v_{Z}\left(\left[\rho^{(\operatorname{tar})}\right] ;(\mathbf{1}) 5\right) \\
\quad+v_{\mathrm{H}}\left(\left[\rho^{(\operatorname{tar})}\right] ; \mathbf{r}\right)+v_{X C}^{(n)}\left(\left[\rho^{n-1}\right] ; \mathbf{r}\right)+v_{X C}\left(\left[\rho^{(\operatorname{tar})}\right] ; \mathbf{r}\right)-\mu^{(\operatorname{tar})}
\end{gathered}
$$

or equivalently, Eq. 9. Thus, the proposed algorithm is derived from the sum of the unknown target equation, cf. Eq. 12 and the Euler equation of the actual density, cf. Eq. 13, iteratively approaching the target density. In Fig. 1 a pictorial depiction of the algorithm is shown. Imagine an ordered list of exchange-correlation potentials $v_{X C}^{(n)}$ each of them yielding a set of eigenfunctions which determine the Pauli potential and the resulting trial density, represented by a straight arrow. The list is supposed to be ordered according to the distance from the desired target density, shown on top of the pictorial scheme. The exchange-correlation potential $v_{X C}^{(1)}$ yields the density $\rho^{(1)}$. Placing $v_{\mathrm{P}}^{(1)}$ in the Euler equation for the target density, cf. Eq. 9 yields a new exchange-correlation potential $v_{X C}^{2}$ which yields a density $\rho^{(2)}$ approaching the desired target density $\rho^{(\operatorname{tar})}$.

For a convex functional the algorithm is guaranteed to converge. In case of degeneracies due to symmetry this assured if the ensemble functional is employed. In that case the Pauli potential is evaluated from:

$\nu_{\mathrm{P}}^{\mathrm{ens}}([\rho] ; \mathbf{r})=\frac{\tau^{\mathrm{ens}}(\mathbf{r})-t_{\mathrm{W}}(\mathbf{r})}{\rho(\mathbf{r})}+\sum_{i}^{\mathrm{occ}} n_{i}\left(\mu-\varepsilon_{i}\right) \frac{\left|\Phi_{i}(\mathbf{r})\right|^{2}}{\rho(\mathbf{r})}$

with:

$\tau^{\mathrm{ens}}(\mathbf{r}) s=1 / 2 \sum_{i} n_{i}\left|\nabla \Phi_{i}(\mathbf{r})\right|^{2}$

and:

$n_{i}= \begin{cases}1 & \text { for } \varepsilon_{i}<\mu \\ 0 & \text { for } \varepsilon_{i}>\mu \\ \in[0,1] & \text { for } \varepsilon_{i}=\mu .\end{cases}$

\section{COMPUTATIONAL DETAILS}

Whereas the algorithm proposed in the Theory Section is general and can be implemented in any convenient molecular or solid state program (as long as the KS eigenfunctions are available), the numerical procedure described in the following applies to spherical systems. For each trial KS potential the radial eigenfunctions are solved numerically on
Fig. 1 A pictorial depiction of the proposed algorithm how to obtain the KS potential for a given target density. A trial exchange potential is chosen determining the corresponding Pauli potential and trial density via its eigenfunctions. The resulting Pauli potential is used to obtain a new exchange-correlation potential via the Euler equation, while all remaining density-based potentials are evaluated from the target density.

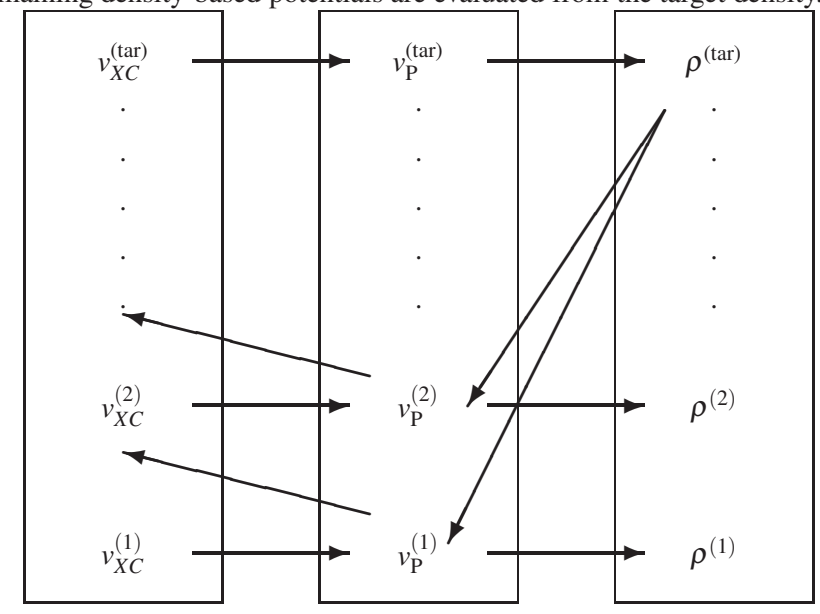

an equidistant mesh with fixed boundary conditions using the Eigen software[33]. All real space properties, like electron density, required potentials, kinetic energy densities and corresponding energies, are evaluated subsequently by own code. Independent data checking was performed with DGrid [34]. The desired spin-polarization of the KS system can be manipulated by the occupation numbers. Adjusting eigenvalues during the iterative procedure in order to manipulate the limiting value of the potential for large radial distances is not needed for the proposed method.

\section{RESULTS AND DISCUSSION}

The proposed algorithm was first tested for a well-known example, the Ne atom. The computation was performed for a distance of 0-4 bohr on an equidistant mesh with 0.001 bohr. Convergence was obtained up to $d_{1}=0.13$ electrons within 35 iterations. Fig. 2 depicts the exchange-correlation potential generated from the HF electron density calculated from the Clementi-Roetti HF wavefunctions [35]. The characteristic oscillation of the KS exchange-correlation potential $v_{X C}^{\mathrm{KS}}$ is located around 0.29 bohr. Sometimes, those oscillations are attributed to the atomic shell structure. Clearly, the number of oscillations coincides with the number of shell separators for a given atom, in case of the $\mathrm{Ne}$ atom $v_{X C}^{\mathrm{KS}}$ exhibits one maximum at 0.29 bohr in close proximity to the ideal shell boundary [36] of 0.27 bohr separating the first and the second atomic shell. Despite that, those oscillations of $v_{X C}^{\mathrm{KS}}$ are by far not responsible for the atomic shell structure, but only a consequence due to the mimicry of the KS system to the real interacting system of interest. 
Fig. 2 Exchange-correlation potential $v_{X C}^{\mathrm{KS}}$ (shown in black) and the Slater potential (shown in red) for the HF electron density of the $\mathrm{Ne}$ atom. The corresponding Pauli potential evaluated from the KS and HF eigenfunctions are depicted by black and red dashed lines respectively. The sum of both, the exchange and the Pauli potential, the so-called Fermi potential, is the same for the non-interacting KS system (shown by the green dashed line) and the interacting HF system (shown by the dark green dashed line).

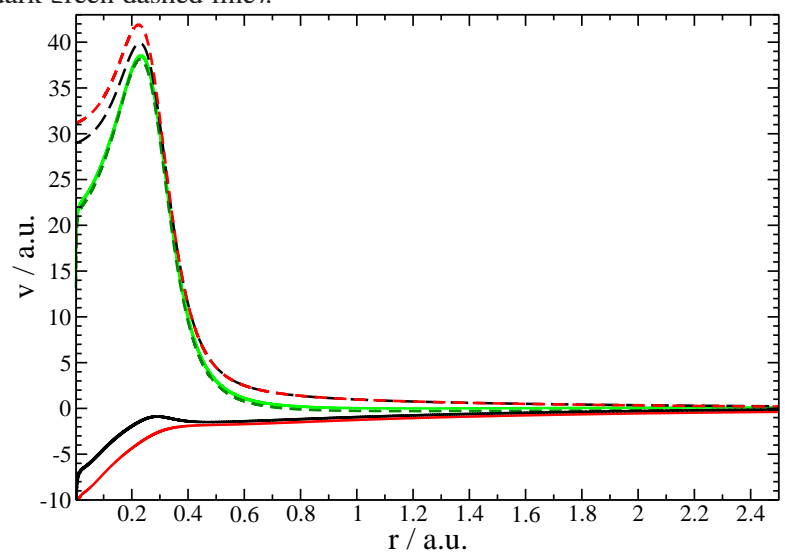

To see that, recall that for the interacting system the exact exchange potential yielding the HF electron density is the Slater potential $v_{X}^{\mathrm{S}}$ [37-39]. The Slater potential, however, is monotone increasing and does not exhibit oscillations at the shell boundaries, see Fig. 2 where $v_{X}^{\mathrm{S}}$ shown in red and $v_{X C}^{\mathrm{KS}}$ shown in black are opposed to one another. Both exchange potentials $\left(v_{X}^{\mathrm{S}}\right.$ and $v_{X C}^{\mathrm{KS}}$ ) yield the HF electron density from Levy-Perdew-Sahni (LPS) formalism [40], which is a second order differential equation for the electron density itself (not for a set of single-particle equations) with the help of an effective one-particle operator. This effective operator contains the so-called Fermi potential [39? ], the sum of the Pauli potential and the exchange potential, cf. Eq. 6. The Fermi potential is purely density dependent, at least at the solution point it can trivially be obtained by inversion of Eq. 6. Within the LPS formalism, $v_{X}^{\mathrm{S}}$ in connection with $v_{\mathrm{P}}$ evaluated from HF eigenfunctions, and $v_{X C}^{\mathrm{KS}}$ in connection with $v_{\mathrm{P}}$ evaluated from KS eigenfunctions, both yield the same density, namely the HF electron density. Therefore, the difference between both exchange potentials only is due to the additional requirement that for the KS system the eigenfunctions have to obey Eq. 1, which is not a requirement for the LPS formalism itself, but does not have additional implications for the physical system and thus, the oscillatory behavior of $v_{X C}^{\mathrm{KS}}$ is not responsible or the atomic shell structure. The characteristic atomic shell structure of the radial electron density is induced by the peaks in the Pauli potential shown by the dashed lines (red HF, black KS) in Fig. 2. For a more detailed discussion see reference [?].

Next, the algorithm was applied to an electron density from a fictitious gedanken experiment. Is it possible to generate such an exchange-correlation potential that the resul- tant KS atomic density exhibits a bosonic-like, structureless radial electron density? Recall that the opposite case, obtaining structureless electron densities from approximate kinetic energy functionals, is a well-known problem in orbitalfree density functional theory. Here, a simple exponential gedanken density was taken $\rho(\mathbf{r})=N \alpha^{3} /(8 \pi) e^{-\alpha r}$. Such gedanken densities for two and four electrons were first studied by Colonna and Savin $[16,17]$. The numerical procedure was carried out for $\alpha=10$ and the density normalized to $N=10$ electrons on a distance of $0-1.5$ bohr with an equidistant mesh of 0.0005 bohr. Convergence was reached with $d_{1}\left(\rho^{(n)}, \rho^{(\operatorname{tar})}\right)=0.01$ electrons within 20 and 45 iterations for the spin-unpolarized and the spin-polarized system, respectively.

As first test case, an Aufbau resembling to the Ne atom was chosen, occupying the orbital set $\left\{1 s^{2}, 2 s^{2}, 2 p^{6}\right\}$. Afterwards, the same gedanken density was regarded as electron density for the spin-polarized state $\left\{\alpha: 1 s^{1}, 2 s^{1}, 2 p^{3}, 3 s^{1}, 3 p^{1}\right\}$ ; $\left\{\beta: 1 s^{1}, 2 s^{1}, 2 p^{1}\right\}$. The algorithm handles both test cases equally well (no special implementation is needed), since the spin-polarization is simply introduced by the corresponding occupation numbers of the resulting eigenfunctions. The exchange-correlation potentials for the chosen gedanken density for the two gedanken states are depicted in Fig. 3. The corresponding effective potentials are shown in Fig. 4, respectively. In both cases all ten electrons are bound. The corresponding eigenvalues, shown by the dashed lines in Fig. 4, are negative for both species. Notice, the reversal of the $\mathrm{s}$ and $\mathrm{p}$ eigenvalues in the Figure (also found by Colonna and Savin for their shell-less electron density models [16]).

Figs. 5 and 6 depict the final gedanken density and its orbital resolved components for the spin unpolarized system and the spin-polarized system, respectively. Obligatorily, all orbitals keep their usual nodal structure (the algorithm solves numerically for the KS eigenfunctions). However, the final electron density is a single exponential and thus, the corresponding radial electron density only exhibits a single maximum (instead of two maxima for a normal atomic density for the $\mathrm{Ne}$ atom). Despite this unusual requirement, the density difference between the targeted electron density $\rho^{(\operatorname{tar})}(\mathbf{r})=N \alpha^{3} /(8 \pi) e^{-\alpha r}$ and the final electron density obtained with the proposed algorithm is surprisingly small with 0.01 electrons for both cases. The density difference as a function of distance is shown in Fig. 7; the error is never very large.

\section{CONCLUSIONS}

A new and simple algorithm for the Kohn-Sham inversion problem was presented. The method is found to converge to a close $v$-representable electron density from the chosen target density irrespective of the fact whether the target den- 
Fig. 3 Exchange-correlation potentials $v_{X C}^{K S}$ for the gedanken density $\rho(\mathbf{r})=N \alpha^{3} /(8 \pi) e^{-\alpha r}$ with $\alpha=10$ and $N=10$ for the unpolarized state $\left\{1 s^{2}, 2 s^{2}, 2 p^{6}\right\}$ (shown in black) and the spin-polarized state $\{\alpha$ : $\left.1 s^{1}, 2 s^{1}, 2 p^{3}, 3 s^{1}, 3 p^{1} ; \beta: 1 s^{1}, 2 s^{1}, 2 p^{1}\right\}$ (shown in red).

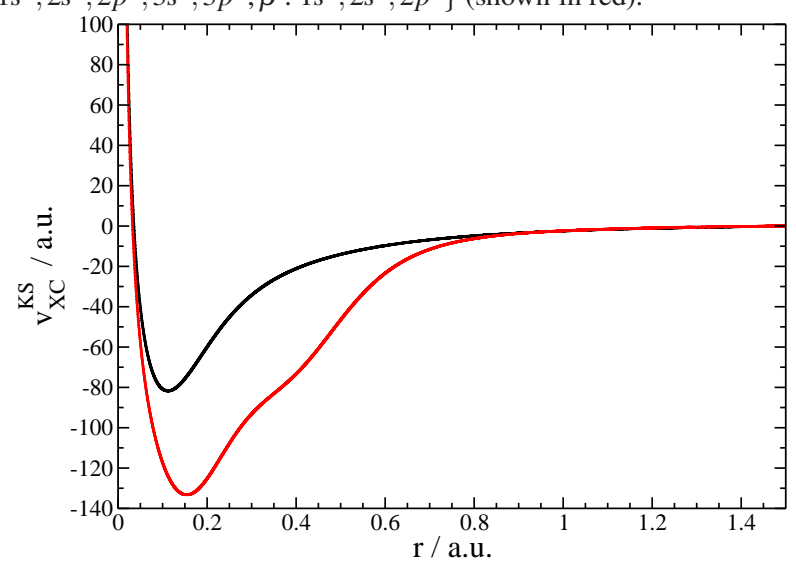

Fig. 4 Effective potentials $v_{\mathrm{eff}}([\rho] ; \mathbf{r})$ for the gedanken density $\rho(\mathbf{r})=N \alpha^{3} /(8 \pi) e^{-\alpha r}$ with $\alpha=10$ and $N=10$ for the unpolarized state $1 s^{2}, 2 s^{2}, 2 p^{6}$ (shown in black) and the spin-polarized state $\left\{\alpha: 1 s^{1}, 2 s^{1}, 2 p^{3}, 3 s^{1}, 3 p^{1} ; \beta: 1 s^{1}, 2 s^{1}, 2 p^{1}\right\}$ (shown in red) and eigenvalues (shown by dashed lines in the same color code). Notice the inversion of the $\mathrm{s}$ and $\mathrm{p}$ eigenvalues.

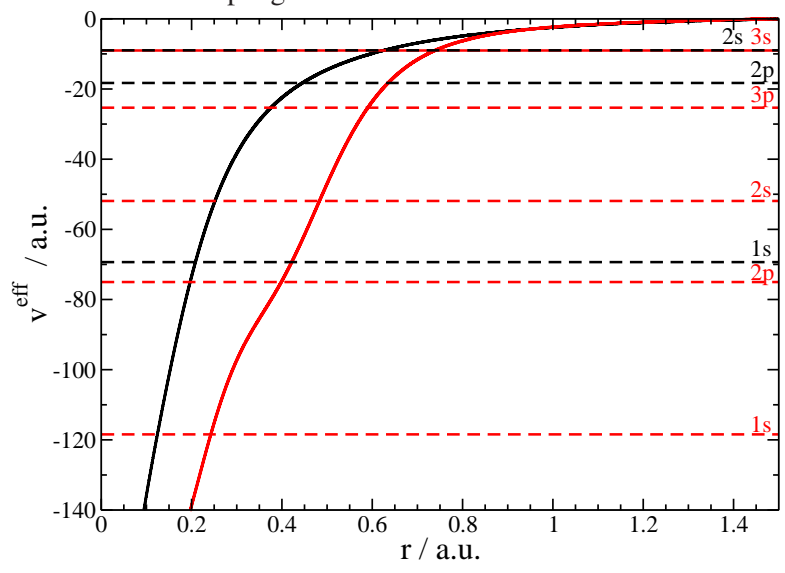

Fig. 5 Gedanken density $\rho(\mathbf{r})=N \alpha^{3} /(8 \pi) e^{-\alpha r}$ with $\alpha=10$ and $N=10$ and its orbital resolved components for the unpolarized state $1 s^{2} \cdot 2 s^{2} \cdot 2 p^{6}$

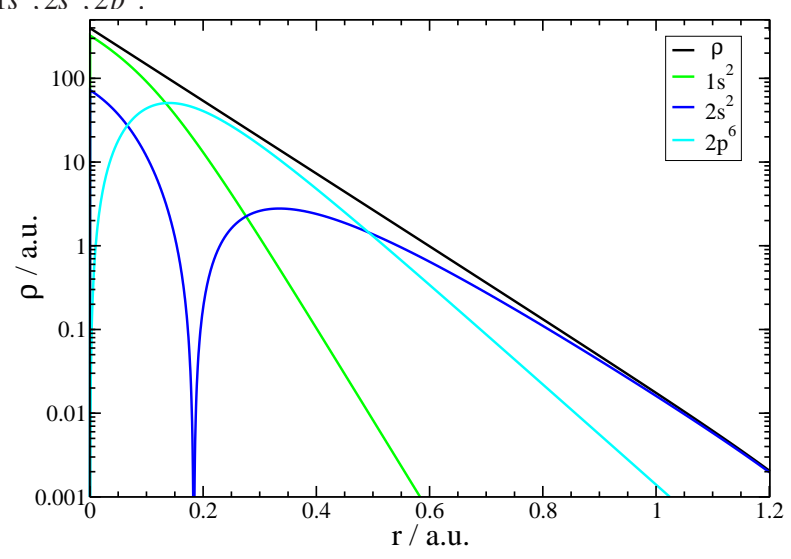

Fig. 6 Gedanken density $\rho(\mathbf{r})=N \alpha^{3} /(8 \pi) e^{-\alpha r}$ with $\alpha=10$ and $N=$ 10 and its orbital resolved components for the spin-polarized state $\{\alpha$ : $\left.1 s^{1}, 2 s^{1}, 2 p^{3}, 3 s^{1}, 3 p^{1} ; \beta: 1 s^{1}, 2 s^{1}, 2 p^{1}\right\}$.

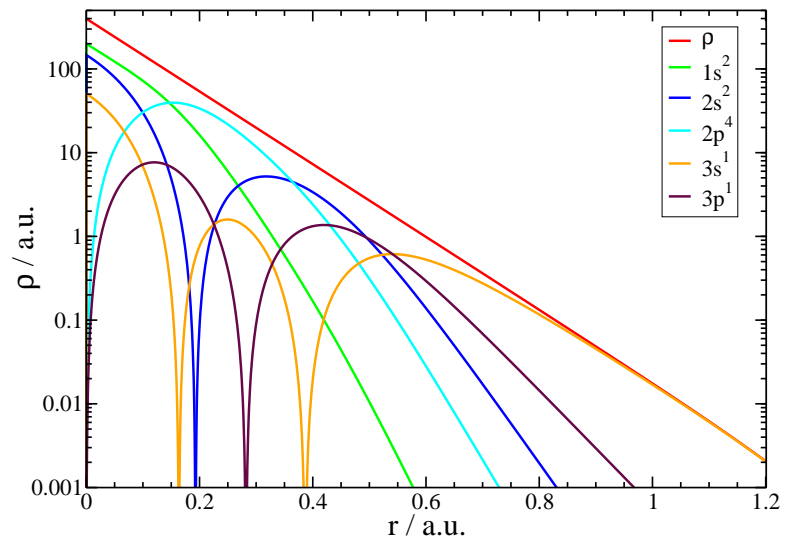

Fig. 7 Density difference between the targeted gedanken density $\rho(\mathbf{r})=N \alpha^{3} /(8 \pi) e^{-\alpha r}$ with $\alpha=10$ and $N=10$ and the final converged electron density for the unpolarized state $1 s^{2}, 2 s^{2}, 2 p^{6}$ (shown in black) and the spin-polarized state $\left\{\alpha: 1 s^{1}, 2 s^{1}, 2 p^{3}, 3 s^{1}, 3 p^{1} ; \beta\right.$ : $\left.1 s^{1}, 2 s^{1}, 2 p^{1}\right\}$ (shown in red).

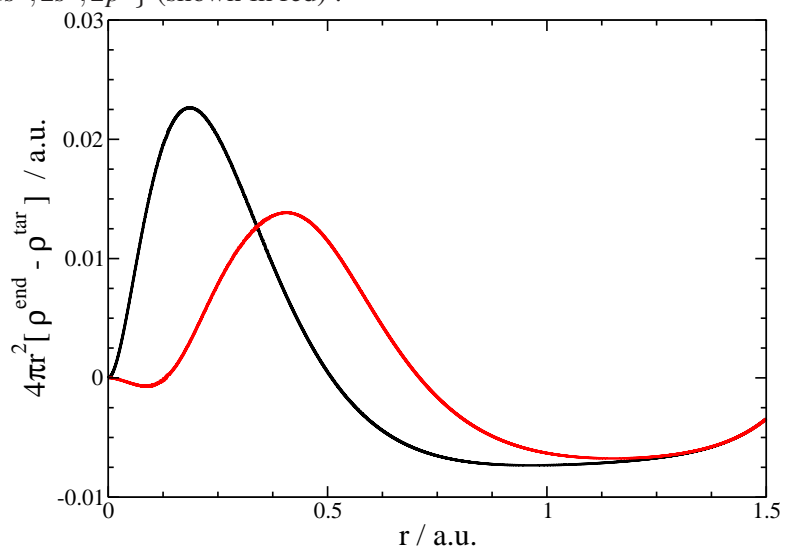

sity was $v$-representable or not. The target density is of general nature. The algorithm handles Hartree-Fock and post Hartree-Fock wavefunction based densities for unpolarized as well as polarized states, but also experimental densities and even gedanken densities can be treated. The method is easy to implement and does not require an additional routine adjusting the KS eigenvalues during the iterative procedure.

The method was applied to a single-exponential gedanken density for a Ne-like atom exhibiting no radial shell structure. It was shown that the resulting potential is able to bind all ten electrons for the chosen spin-polarized and unpolarized test cases.

\section{ACKNOWLEDGMENTS}

K.F. wishes to thank Dr. Miroslav Kohout for fruitful discussions and substantial encouragement over years. The au- 
thors gratefully acknowledge Dr. Stijn De Baerdemaecker for valuable comments and discussions on the numerical procedure. Dr. Guillaume Acke is acknowledged for valuable help concerning the Eigen software. The authors gratefully acknowledge the Fund for Scientific Research in Flanders (FWO-Vlaanderen) for research grant G021115N.

\section{References}

1. Dreizler RM, Gross EKU (1990) Density Functional Theory. Springer Verlang, Berlin Heidelberg

2. Parr RG, Yang W (1989) Density-Functional Theory of Atoms and Molecules. Oxford University Press

3. Hohenberg P, Kohn W (1964) Phys Rev B 136:864-871

4. Talman JD, Shadwick WF (1976) Phys Rev A 14:36-40

5. Nagy A, March N (1989) Phys Rev A 39:5512-5514

6. Holas A, March N (1991) Phys Rev A 44:5521

7. Wang Y, Parr RG (1993) Phys Rev A 47:1591-1593

8. Görling A (1992) Phys Rev A 46:3753-3757

9. Zhao Q, Parr RG (1993) J Chem Phys 98:543-548

10. Zhao Q, Morrison RC, Parr RG (1994) Phys Rev A 50:2138-2142

11. Görling A, Levy M (1994) Phys Rev A 50:196-204

12. van Leeuwen R, Baerends EJ (1994) Phys Rev A 49:2421-2431

13. Görling A, Levy M (1995) Phys Rev B 53:7024-7029

14. Tozer DJ, Ingamells VE, Handy NC (1996) J Chem Phys 105:9200-9213

15. Tozer DJ, Handy WH N C aand Green (1997) Chem Phys Lett 273:183-194

16. Colonna F, Savin A (1999) J Chem Phys 110:28282835

17. Pollet R, Colonna F, Leininger H T aand Stoll, Werner HJ, Savin A (1999) J Chem Phys 110:2828-2835

18. Yang W, Wu Q (2002) Phys Rev Lett 89:143002

19. Wu Q, Yang W (2003) J Chem Phys 118:2498-2509

20. Peirs K, Van Neck D, Waroquier M (2003) Phys Rev A 67:012505

21. Teale AM, Coriani S, Helgaker T (2009) J Chem Phys 130:104111

22. Teale AM, Coriani S, Helgaker T (2010) J Chem Phys 132:164115

23. Ryabinkin IG, Kananenka AA, Staroverov VN (2013) Phys Rev Lett 111:074112

24. Ospadov E, Ryabinkin IG, Staroverov VN (2017) J Chem Phys 146:084103

25. Perdew JP, Ruzsinszky A, Sun J, Burke K (2014) J Chem Phys 140:18A533

26. Kohn W, Sham LJ (1965) Phys Rev A 140:1133-1138

27. von Weizsäcker CF (1935) Z Phys 96:431-458

28. March NH (1986) Phys Lett A 113:476-478

29. Levy M, Ou-Yang H (1988) Phys Rev A 38:625-629

30. Fermi E, Amaldi E (1934) AccadItalRome 6:117
31. Ayers PW, Morrison RC, Parr RG (2005) Mol Phys 103:2061-2072

32. Finzel K, Grin Y, Kohout M (2012) Theor Chem Acc 131:1106

33. Guennebaud G, Jacob B, et al (2010) Eigen v3. http://eigen.tuxfamily.org

34. Kohout M (2016) DGrid, version 5.0. Dresden

35. Clementi E, Roetti C (1974) At Data Nucl Data Tables $14: 177-478$

36. Schmider H, Sagar R, Smith Jr VH (1992) Can J Chem 70:506-512

37. Slater JC (1951) Phys Rev 81:385-390

38. Finzel K (2016) Int J Quantum Chem 116:1187-1189

39. Finzel K, Ayers PW (2017) Int J Quantum Chem DOI: 10.1002/qua. 25364

40. Levy M, Perdew JP, Sahni V (1984) Phys Rev A $30: 2745-2748$ 